\title{
Mechanisms of triboluminescence
}

\author{
(radiationless transitions/electric field effect/pressure effect)
}

\section{S. H. LIN*, D. WUTZ*, Z. Z. HO*, AND H. EYRING ${ }^{\dagger}$}

${ }^{*}$ Department of Chemistry, Arizona State University, Tempe, Arizona 85281; and ${ }^{\dagger}$ Department of Chemistry, University of Utah, Salt Lake City, Utah 84112

Contributed by Henry Eyring, January 2, 1980

\begin{abstract}
The purpose of this paper has been to develop the theoretical treatment of the triboexcitation mechanisms due to the electric field effect and the pressure effect and to show how to analyze the triboluminescent spectra to determine the external factors that affect or induce the triboluminescence.
\end{abstract}

The emission of light caused by application of mechanical energy to a solid is usually called triboluminescence (TL). Although the detailed assignments of excited-state origins and spectral features of TL are possible due to recent advances in TL spectroscopy (1), the mechanisms by which TL is excited are not well understood. The three mechanisms of triboexcitation that are currently under active consideration can be broadly classified as electrical, thermal, and chemical (1). It is the purpose of this paper to study theoretically the first two mechanisms-i.e., the triboexcitation due to the electric effect and that due to the high pressure effect. The last triboexcitation mechanism that involves the production of the excited electronic state by a chemical reaction induced by the mechanical energy will not be discussed here. It should be noted that TL might play a very important role in detonations in condensed explosives.

\section{Theory}

In this section, we shall present the theoretical treatment of the triboexcitations due to the high pressure effect and the electric field effect. In other words, we have to consider the mechanisms of the population of the excited electronic states by radiationless transitions induced or enhanced by the electric field effect and the high pressure effect.

According to the conventional theory of radiationless transitions, the thermal average rate constant of the radiationless transition $\mathrm{b} \rightarrow \mathrm{a}$ can be expressed as (2)

$$
W_{b a}=\frac{2 \pi}{\hbar} \sum_{v^{\prime}} \sum_{v^{\prime \prime}} P_{b v^{\prime}}\left|\left\langle\Psi_{a v^{\prime \prime}}\left|\hat{H}_{B o}^{\prime}\right| \Psi_{b v^{\prime}}\right\rangle\right|^{2} \delta\left(E_{b v^{\prime}}-E_{a v^{\prime \prime}}\right) .
$$

In the presence of external variables such as the electric field $F$, the magnetic field $H$, and the high pressure $P$, the rate of radiationless transitions will be affected as a result of the effect of these external variables on the wavefunction and energy and on the opening of the new nonradiative channel (3).

We first consider the electric field effects. In this case, eq 1 becomes (4)

$$
\begin{aligned}
& W_{b a}(F)=\frac{2 \pi}{\hbar} \sum_{v^{\prime}} \sum_{v^{\prime \prime}} P_{b v^{\prime}}(F) \\
& \times\left|\left\langle\Psi_{a v^{\prime \prime}}(F)\left|\hat{H}^{\prime}\right| \Psi_{b v^{\prime}}(F)\right\rangle\right|^{2} \delta\left[E_{a v^{\prime \prime}}(F)-E_{b v^{\prime}}(F)\right],
\end{aligned}
$$

where

$$
\hat{H}^{\prime}=\hat{H}_{B o}^{\prime}+\hat{H}_{F}^{\prime}
$$

The publication costs of this article were defrayed in part by page charge payment. This article must therefore be hereby marked "advertisement" in accordance with 18 U. S. C. $\$ 1734$ solely to indicate this fact. and $\hat{H}^{\prime}{ }_{F}$ represents the off-diagonal portion of the Stark interaction. In the weak field case, the perturbation method can be used and the treatment is similar to the magnetic field effect (3). For a system of randomly oriented molecules, the first-order terms disappear and we have

$$
W_{b a}(F)=W_{b a}+F^{2} \sum_{n=1}^{6} W_{b a}^{(2)}(n)+\ldots,
$$

where $W_{b a}$ represents the rate constant in the absence of the electric field and $W_{b a}^{(2)}(n)$ are defined by

$$
\begin{aligned}
& W_{b a}^{(2)}(1)=\frac{2 \pi}{3 \hbar} \sum_{v^{\prime \prime}} \sum_{v^{\prime}} P_{b v^{\prime}}\left|\vec{R}_{a v^{\prime \prime}, b v^{\prime}}\right|^{2} \delta\left(E_{b v^{\prime}}-E_{a v^{\prime \prime}}\right), \\
& W_{b a}^{(2)}(2)=-\frac{4 \pi \beta}{3 \hbar} \sum_{v^{\prime \prime}} \sum_{v^{\prime}} P_{b v^{\prime}} \\
& \times \operatorname{Re}\left[\left(\vec{R}_{a v^{\prime \prime}, b v^{\prime}} \cdot \vec{R}_{b v^{\prime}, b v^{\prime}}\right)\left\langle\Psi_{b v^{\prime}}\left|\hat{H}_{B o}^{\prime}\right| \Psi_{a v^{\prime \prime}}\right\rangle\right] \\
& \times \delta\left(E_{b v^{\prime}}-E_{a v^{\prime \prime}}\right) \text {, } \\
& W_{b a}^{(2)}(3)=-\frac{4 \pi}{3 \hbar} \sum_{v^{\prime \prime}} \sum_{v^{\prime}} P_{b v^{\prime}} \operatorname{Re}\left[\left\{\vec{R}_{a v^{\prime \prime}, b v^{\prime}} \cdot \Delta \vec{R}\left(a v^{\prime \prime}, b v^{\prime}\right)\right\}\right. \\
& \left.\left\langle\Psi_{b v^{\prime}}\left|\hat{H}_{B o}^{\prime}\right| \Psi_{a v^{\prime \prime}}\right\rangle\right] \delta^{\prime}\left(E_{b v^{\prime}}-E_{a v^{\prime \prime}}\right), \\
& W_{b a}^{(2)}(4)=\frac{\pi \beta}{3 \hbar} \sum_{v^{\prime \prime}} \sum_{v^{\prime}} P_{b v^{\prime}} T_{a v^{\prime \prime}, b v^{\prime}}\left[\operatorname{Tr}\left\{\bar{\alpha}\left(b v^{\prime}\right)\right\}-\left\langle\operatorname{Tr}\left\{\overline{\bar{\alpha}}\left(b v^{\prime}\right)\right\}\right\rangle\right. \\
& \left.+\beta\left(\left|\vec{R}_{b v^{\prime} b v^{\prime}}\right|^{2}-\left\langle\left|\vec{R}_{b v^{\prime} b v^{\prime}}\right|^{2}\right\rangle\right)\right] \delta\left(E_{b v^{\prime}}-E_{a v^{\prime \prime}}\right), \\
& W_{b a}^{(2)}(5)=\frac{2 \pi}{3 \hbar} \sum_{v^{\prime \prime}} \sum_{v^{\prime}} P_{b v^{\prime}} T_{a v^{\prime \prime}, b v^{\prime}}\left[\beta \vec{R}_{b v^{\prime}, b v^{\prime}} \cdot \Delta \vec{R}\left(a v^{\prime \prime}, b v^{\prime}\right)\right. \\
& \left.+\frac{1}{2} \operatorname{Tr}\left\{\Delta \overline{\bar{\alpha}}\left(a v^{\prime \prime}, b v^{\prime}\right)\right\}\right] \delta^{\prime}\left(E_{b v^{\prime}}-E_{a v^{\prime \prime}}\right),
\end{aligned}
$$

and

$$
\begin{aligned}
W_{b a}^{(2)}(6)=\frac{\pi}{3 \hbar} \sum_{v^{\prime \prime}} \sum_{v^{\prime}} P_{b v^{\prime}} T_{a v^{\prime \prime}, b v^{\prime}}\left|\Delta \vec{R}\left(a v^{\prime \prime}, b v^{\prime}\right)\right|^{2} & \\
& \times \delta^{\prime \prime}\left(E_{b v^{\prime}}-E_{a v^{\prime \prime}}\right),
\end{aligned}
$$

where $T_{a v^{\prime \prime}, b v^{\prime}}=\left|\left\langle\Psi_{a v^{\prime \prime}}\left|\hat{H}_{B o}^{\prime}\right| \Psi_{b v^{\prime}}\right\rangle\right|^{2}$ and $\vec{R}_{a v^{\prime \prime}, b v^{\prime}}=$ $\left\langle\Psi_{a v^{\prime \prime}}|\vec{R}| \Psi_{b v^{\prime}}\right\rangle$, the matrix element of the electric moment.

As in the case of the magnetic field effect on radiationless transitions (3), the term $F^{2} W_{b a}^{(2)}(1)$ will be called the electric field-induced radiationless transition. Other terms $\left[W_{b a}^{(2)}(2)-\right.$ $\left.W_{b a}^{(2)}(6)\right]$ result from the Strek effect on the energy levels of the system. In other words, in Eqs. 5-10 the electric field effect on the wavefunction has been ignored; this point has been discussed recently by Strek (5).

At $T=0, W_{b a}^{(2)}(2)$ and $W_{b a}^{(2)}(4)$ disappear and we have

$$
\begin{gathered}
W_{b a}^{(2)}(1)=\frac{2 \pi}{3 \hbar} \sum_{v^{\prime \prime}}\left|\vec{R}_{a v^{\prime \prime}, b o}\right|^{2} \delta\left(E_{b o}-E_{a v^{\prime \prime}}\right), \\
W_{b a}^{(2)}(3)=-\frac{4 \pi}{3 \hbar} \sum_{v^{\prime \prime}} \operatorname{Re}\left[\left\{\vec{R}_{a v^{\prime \prime}, b o} \cdot \Delta \vec{R}\left(a v^{\prime \prime}, b o\right)\right\}\right. \\
\left.\times\left\langle\Psi_{b o}\left|\hat{H}_{B o}^{\prime}\right| \Psi_{a v^{\prime \prime}}\right\rangle\right] \delta^{\prime}\left(E_{b o}-E_{a v^{\prime \prime}}\right),
\end{gathered}
$$

Abbreviation: TL, triboluminescence. 
$W_{b a}^{(2)}(5)=\frac{\pi}{3 \hbar} \sum_{v^{\prime \prime}} T_{a v^{\prime \prime}, b o} \operatorname{Tr}\left[\Delta \overline{\bar{\alpha}}\left(a v^{\prime \prime}, b o\right)\right] \delta^{\prime}\left(E_{b o}-E_{a v^{\prime \prime}}\right)$,

and

$$
W_{b a}^{(2)}(6)=\frac{\pi}{3 \hbar} \sum_{v^{\prime \prime}} T_{a v^{\prime \prime}, b o}\left|\Delta \vec{R}\left(a v^{\prime \prime}, b o\right)\right|^{2} \delta^{\prime \prime}\left(E_{b o}-E_{a v^{\prime \prime}}\right) .
$$

How to simplify $W_{b a}$ and $W_{b a}^{(2)}(n)$ is well known $(2,4,5)$. For example, let us compare $W_{b a}$ and $F^{2} W_{b a}^{(2)}(1)$ at $T=0$; by using the Einstein model we find

$$
\frac{F^{2} W_{b a}^{(2)}(1)}{W_{b a}}=\frac{\frac{2}{3}\left|\vec{R}_{a b}\right|^{2} F^{2}}{\frac{\omega_{i}^{\prime}}{\hbar}\left|R_{i}(a b)\right|^{2}} \cdot \frac{\omega^{\prime}}{\omega_{b a}},
$$

where $\vec{R}_{a b}=\left\langle\Phi_{a}|\vec{R}| \Phi_{b}\right\rangle$, the electronic transition moment $R_{i}$ $=-\hbar^{2}\left\langle\Phi_{a}\left|\partial / \partial Q_{i}\right| \Phi_{b}\right\rangle, \omega_{b a}$ represents the energy gap, and $\omega_{i}{ }^{\prime}$ and $\omega^{\prime}$ are vibrational frequencies.

It should be noted that the electrical mechanisms that have been proposed so far all require that mechanical energy generates free electrons, which leads to emission of a photon by electron impact on molecules, electroluminescence, or recombination of cations and anions. In this paper, we propose another triboexcitation mechanism due to the electric fieldinduced radiationless transition; the electric field can in one way arise from a piezoelectric polarization.

Next we consider the triboexcitation due to the pressureinduced radiationless transition. In this case, Eq. 2 becomes

$$
\begin{aligned}
& W_{b a}(P)=\frac{2 \pi}{\hbar} \sum_{v^{\prime}} \sum_{v^{\prime \prime}} P_{b v^{\prime}}(P) \\
& \quad \times\left|\left\langle\Psi_{a v^{\prime \prime}}(P)\left|\hat{H}^{\prime}\right| \Psi_{b v^{\prime}}(P)\right\rangle\right|^{2} \delta\left[E_{a v^{\prime \prime}}(P)-E_{b v^{\prime}}(P)\right],
\end{aligned}
$$

where $\hat{H}^{\prime}=\hat{H}_{B o}^{\prime}+\hat{H}_{p}^{\prime}$. In other words, we replace the electric field $F$ by the pressure $P$. $\hat{H}_{p}^{\prime}$ is given by (6)

$$
\hat{H}_{p}^{\prime}=\sum_{\alpha}\left(\frac{\partial \hat{H} e}{\partial q_{\alpha}}\right)_{o} q_{\alpha}+\ldots
$$

where $\hat{H} e$ represents the Hamiltonian of electronic motion and $q_{\alpha} s$ represent the nuclear coordinate displacements induced by external pressure, $P$. When the external pressure is not extremely high, the nuclear displacements $q_{\alpha}$ will be linearly related to $P$ and the corresponding instantaneous nuclear coordinate,

$$
q_{\alpha}=P \beta_{\alpha}\left(R_{\alpha}^{o}+\Delta R_{\alpha}\right)=P \beta_{\alpha}\left(R_{\alpha}^{o}+\sum_{j} a_{\alpha j} Q_{j}{ }^{\prime \prime}\right),
$$

where $\beta_{\alpha}$, represents the linear compressibility modulus, $R_{\alpha}{ }^{\circ}$ is the equilibrium nuclear coordinate, and $\Delta R_{\alpha}$ represents the small displacement of the nuclear coordinate from its equilibrium position during vibration, which in turn can be expressed in terms of normal coordinates $Q_{j}{ }^{\prime \prime}$ of the ground electronic state.

By using Eqs. 17 and 18, we can find the explicit expression of $W_{b a}(P)$ as a function of $P$ in the same manner as that presented in Eqs. 2-15. For example, the pressure-induced nonradiative rate constant can be expressed as

$$
\begin{aligned}
P^{2} W_{b a}^{(2)}(1)_{p}= & \frac{2 \pi}{\hbar} \sum_{v^{\prime \prime}} \sum_{v^{\prime}} P_{b v^{\prime}} \\
& \times\left|\left\langle\Psi_{a v^{\prime \prime}}\left|H_{p}^{\prime}\right| \Psi_{b v^{\prime}}\right\rangle\right|^{2} \delta\left(E_{a v^{\prime \prime}}-E_{b v^{\prime}}\right) .
\end{aligned}
$$

In other words, in this case the vibronic coupling $\left\langle\Psi_{a v^{\prime \prime}}\left|\left(\partial \hat{H}_{e} / \gamma q_{\alpha}\right)_{o}\right| \Psi_{b v^{\prime}}\right\rangle$ is responsible for determining the nonradiative rate. The expression of the radiationless transition rate constant that describes the high pressure effect on $W_{b a}$ through the changes at energy levels has been given (6).
For triboluminescence, the existence of a specific mechanism is difficult to assess because one or several of them could be simultaneously operative.

\section{Discussion}

In the previous section, we have presented the theoretical treatment of the triboexcitation mechanisms due to the electric field effect and high pressure effect. In this section, we shall attempt to analyze the triboluminescence of sugars. Recently Zink et al. (7) have spectroscopically investigated the TL of mono- and oligosaccharides. They have found that samples of D-glucose, lactose, maltose, L-rhamnose, and sucrose are triboluminescent, whereas samples of cellobiose, fructose, fucose, galactose, and mannose are not. The TL spectrum of sucrose, a typical member of the triboluminescent sugars, is reproduced in Fig. 1. Zink et al. have attributed the spectrum to the second positive group of molecular nitrogen-i.e., due to the ${ }^{3} \pi_{u} \rightarrow$ ${ }^{3} \pi_{g}$ transition of $\mathbf{N}_{2}$.

We have verified the spectrum shown in Fig. 1 to be that of the second positive group of $\mathrm{N}_{2}$ by calculating the vibrational progressions and showing that they indeed arise from the vibrational levels of the ${ }^{3} \pi_{g}$ state of $\mathrm{N}_{2}(8)$. The assignment of these vibrational progressions is given in Table 1.

In Table 1 are also tabulated the Franck-Condon factors calculated by three methods (A, B, and C). In using the displaced oscillator method, the average vibrational frequency of $N_{2}$ between the ${ }^{3} \pi_{g}$ and ${ }^{3} \pi_{u}$ states has been used. As we can see from Table 1, the three methods give comparable results.

We have resolved the TL spectrum shown in Fig. 1, and the relative intensity of the vibrational bands is given in Table 1 . It should be noted that there exist two hot bands (1-0 and 2-5) in the TL spectrum. No photoluminescent spectrum of the second positive group of $\mathbf{N}_{2}$ in solids has been reported but, from the Franck-Condon factor calculations (see Table 1), we can see that the calculated relative intensity of vibrational bands should be a good representative of the photoluminescent spectrum of the second positive group of $\mathbf{N}_{2}$.

If we compare the TL spectrum and theoretical photoluminescent spectrum given in Table 1 , it can be seen that these two spectra are quite different, although the peak positions of the two spectra are identical. Because the displaced oscillator method can provide a good approximate result for calculating the Franck-Condon factors in this case, we shall use this method to analyze the TL spectrum. The Franck-Condon factor for the

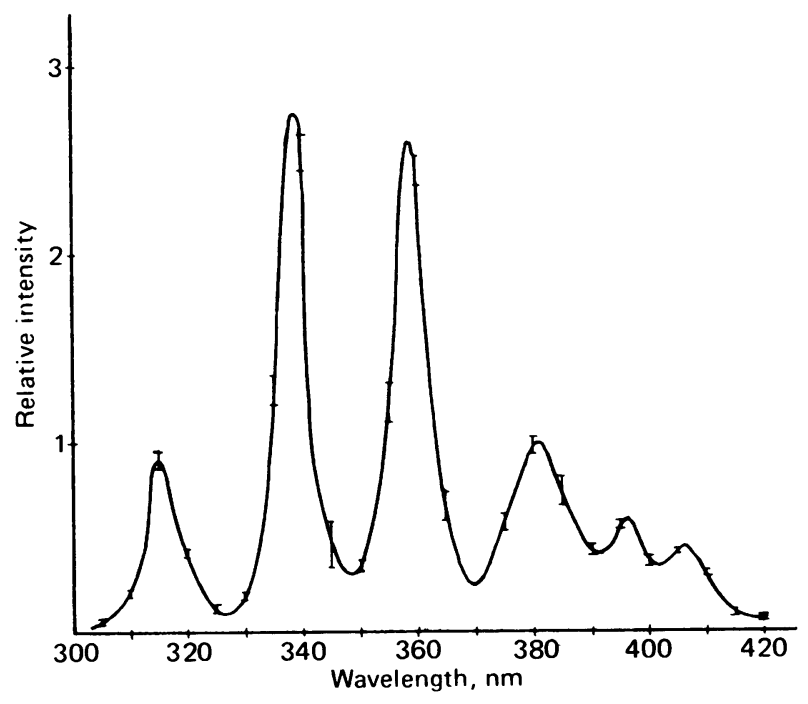

FIG. 1. TL spectrum of sucrose. 
Table 1. Experimental and theoretical results

\begin{tabular}{|c|c|c|c|c|c|c|c|}
\hline \multirow[b]{2}{*}{ Transitions } & \multicolumn{3}{|c|}{ Franck-Condon factor } & \multicolumn{3}{|c|}{ Relative intensity } & \multirow{2}{*}{$\begin{array}{l}\text { Relative intensity } \\
\text { (TL spectrum) }\end{array}$} \\
\hline & A & B & $\mathrm{C}$ & A & B & $\mathrm{C}$ & \\
\hline $1-0$ & 0.331 & 0.36 & 0.33 & 0.73 & 0.80 & 0.74 & 0.35 \\
\hline $0-0$ & 0.455 & 0.450 & 0.448 & 1 & 1 & 1 & 1 \\
\hline $0-1$ & 0.388 & 0.36 & 0.33 & 0.85 & 0.80 & 0.74 & 1.19 \\
\hline $0-2$ & 0.134 & 0.15 & 0.15 & 0.29 & 0.33 & 0.33 & 0.70 \\
\hline $0-3$ & 0.0216 & 0.040 & 0.031 & 0.06 & 0.08 & 0.07 & 0.17 \\
\hline $2-5$ & & & & & & & 0.19 \\
\hline
\end{tabular}

Methods for calculating the Franck-Condon factor: A, Rydberg-Klein-Rees of Zare et al. (9); B, displaced oscillator; $\mathrm{C}$, displaced distorted oscillator.

transition $b o \rightarrow a v^{\prime \prime}$, according to the displaced oscillator method, can be expressed as

$$
F_{b o \rightarrow a v^{\prime \prime}}=\frac{S^{v^{\prime \prime}}}{v^{\prime \prime} !} \exp (-S)
$$

where $S$ is the coupling constant and is related to the normal coordinate displacement between the two electronic states (2).

For the TL spectrum, we can determine the value as $S_{\mathrm{TL}}=$ 1.19 from the 0-0 and 0-1 bands, which should be compared with that $\left(S_{\mathrm{PL}}=0.80\right)$ of the photoluminescent spectrum. By using $S_{\mathrm{TL}}=1.19$ and Eq. 20, we can predict the relative intensity of the 0-2 band as 0.71 in good agreement with the experimental result of 0.70 . The difference between $S_{\mathrm{TL}}=1.19$ and $S_{\mathrm{PL}}=0.80$ can be due to the pressure effect or to the electric field effect. Because $\mathrm{N}_{2}$ is nonpolar, the electric field effect in this case must be small because it can arise only from the normal coordinate dependence of the polarizability of $\mathrm{N}_{2}$ (4). It has been shown in a previous paper (6) that the pressure can affect the coupling constant; the pressure dependence of $S$ in this case can be expressed as $(6,10)$

$$
S_{\mathrm{TL}}=S_{\mathrm{PL}}(1+a P)^{2}
$$

By using $S_{\mathrm{TL}}=1.19$ and $S_{\mathrm{PL}}=0.80$, we find $a P=0.22$.

From the existence of hot bands (1-0 and 2-5), we can conclude that in the triboexcitation the vibrational levels $v^{\prime}=0$, $v^{\prime}=1$, and $v^{\prime}=2$ of the ${ }^{3} \pi_{u}$ state are populated and that the vibrational relaxation in the ${ }^{3} \pi_{u}$ state is slow compared with the emission process. Recent measurements of the vibrational relaxation of $\mathrm{N}_{2}$ in dense media (11) indeed show that the rate of vibrational relaxation of $\mathrm{N}_{2}$ is slow (order of seconds or even longer).
In concluding the discussion, it should be noted that in this paper we have presented the theoretical treatment of the triboexcitation mechanisms due to the electric field effect and pressure effect and have shown how to analyze the TL spectra to determine the external factors that affect the TL spectra. In view of recent rapid development in the experimental techniques in photophysics, one should be able to probe the fate of the excited electronic states created in TL; this type of experimental result will provide us additional information to determine the TL mechanisms.

We acknowledge the support of the U.S. Army Research Office (Grant DAAG2978G0029).

1. Zink, J. I. (1978) Accts. Chem. Res. 11, 289-295.

2. Lin, S. H. (1966) J. Chem. Phys. 44, 3759-3766.

3. Selzle, H. L., Lin, S. H. \& Schlag, E. W. (1979) Chem. Phys. Lett. 62, 230-234.

4. Lin, S. H. (1975) J. Chem. Phys. 62, 4500-4524.

5. Strek, W. (1978) Chem. Phys. Lett. 57, 121-124.

6. Lin, S. H. (1973) J. Chem. Phys. 59, 4458-4467.

7. Zink, J. I., Hardy, G. E. \& Sutton, J. E. (1976) J. Phys. Chem. 80, 248-249.

8. Pearse, R. W. B. \& Gaydon, A. G. (1976) The Identification of Molecular Spectra (Chapman-Hall, London), pp. 219-222.

9. Zare, R. N., Larsson, E. O. \& Berg, R. A. (1965) J. Mol. Spectrosc. 15, 117-139.

10. Drickamer, H. G., Frank, C. W. \& Slichter, C. P. (1972) Proc. Natl. Acad. Sci. USA 69, 933-937.

11. Legay, F. (1978) in Chemical and Biological Applications of Lasers, ed. Moore, C. B. (Plenum, New York), Vol. 3, pp. 6185. 